\title{
Efficacy of seasonal pandemic influenza hemagglutinin DNA vaccines delivered by electroporation against aseasonal H1N1 virus challenge in mice
}

\author{
TAN Lei ${ }^{1,2}$, LU HuiJun ${ }^{1}$, ZHANG Dan $^{1,2}$, WANG KaiYan ${ }^{1,3}$, TIAN MingYao ${ }^{1,2}$, \\ LIU CunXia ${ }^{1,2}$, LIU YanYu ${ }^{1,2}$, HU Bo $^{1,2}$ \& JIN NingYi ${ }^{1 *}$ \\ ${ }^{1}$ Genetic Engineering Laboratory of PLA, Academy of Military Medical Sciences of PLA, Changchun 130062, China; \\ ${ }^{2}$ College of Animal Science and Veterinary Medicine, Jilin University, Changchun 130062, China; \\ ${ }^{3}$ Agricultural College of Yanbian University, Yanji 133400, China
}

Received October 20, 2010; accepted January 19, 2011; published online March 17, 2011

\begin{abstract}
Prophylactic DNA vaccines against the influenza virus are promising alternatives to conventional vaccines. In this study, we generated two candidate gene-based influenza vaccines encoding either the seasonal or pandemic hemagglutinin antigen (HA) from the strains A/New Caledonia/20/99 (H1N1) (pV1A5) and A/California/04/2009 (H1N1) (pVEH1), respectively. After verifying antigen expression, the immunogenicity of the vaccines delivered intramuscularly with electroporation was tested in a mouse model. Sera of immunized animals were tested in hemagglutination inhibition assays and by ELISA for the presence of HA-specific antibodies. HA-specific T-cells were also measured in IFN- $\gamma$ ELISpot assays. The protective efficacy of the candidate influenza vaccines was evaluated by measuring mortality rates and body weight after a challenge with $100 \mathrm{LD}_{50}$ of mouse-adapted A/New Caledonia/20/99 (H1N1). Mice immunized with either one of the two vaccines showed significantly higher $\mathrm{T}$ cell and humoral immune responses $(P<0.05)$ than the pVAX1 control group. Additionally, the pV1A5 vaccine effectively protected the mice against a lethal homologous mouse-adapted virus challenge with a survival rate of $100 \%$ compared with a $40 \%$ survival rate in the pVEH1 vaccinated group $(P<0.05)$. Our study indicates that the seasonal influenza DNA vaccine completely protects against the homologous A/New Caledonia/20/99 virus (H1N1), while the pandemic influenza DNA vaccine only partially protects against this virus.
\end{abstract}

seasonal influenza, pandemic influenza, hemagglutinin, DNA vaccine, electroporation, H1N1 influenza virus

Citation: Tan L, Lu H J, Zhang D, et al. Efficacy of seasonal pandemic influenza hemagglutinin DNA vaccines delivered by electroporation against aseasonal H1N1 virus challenge in mice. Sci China Life Sci, 2011, 54: 293-299, doi: 10.1007/s11427-011-4150-5

A new influenza A subtype H1N1 recently emerged, causing a human pandemic. Vaccination is the most effective measure to control the spread of the virus and to reduce the associated morbidity and mortality. However, existing evidence shows that the formerly used trivalent seasonal influenza vaccines are unlikely to provide protection against the new virus strain. Therefore, the development of new vaccines to protect against this virus is urgently needed.

*Corresponding author (email: jinningyi2000@yahoo.com.cn)
Immunization with inactivated vaccines has long been the main method used for preventing influenza infections. However, approaches other than conventional vaccines have also been found to induce protective immunity against important structural proteins of the H1N1 virus. Promising approaches include recombinant protein vaccines [1], adenovirus-based technologies [2] and DNA plasmids [3]. Plasmid DNA vaccines are non-pathogenic, stable at ambient temperatures, economical to produce, and they can be used for repeated immunizations. Many studies in chicken 
and mice have shown that DNA vaccines can provide protection against influenza subtypes $\mathrm{H} 1, \mathrm{H} 3, \mathrm{H} 5, \mathrm{H} 7$, and H9 [4-8]. In particular, DNA vaccines that express the hemagglutinin (HA) protein can protect mice from the viral challenge of either influenza A or B [9-13]. As well as the choice of vaccine antigen, the method of delivery of the vaccines can also influence their effectiveness. Recent animal studies suggest that DNA vaccines can be delivered by in vivo electroporation (EP), resulting in high transfection efficiency and increased protein expression [14,15]. EP requires the application of short electrical pulses to the target tissue, rendering the cell membrane transiently permeable to DNA and other molecules. The effectiveness of EP as a non-viral gene delivery tool and its capacity for enhancing the potency of DNA vaccines have been demonstrated. Furthermore, EP has been frequently and successfully used to augment the potency of muscle-targeted DNA vaccines.

Heterosubtypic immunity (HSI) is defined as cross-protection against infection with an influenza A serotype other than the one used for primary infection or immunization. HSI is thought to be mediated by serotype cross-reactive cytotoxic $\mathrm{T}$ lymphocytes (CTL) that recognize the conserved epitopes of structural proteins. However, recent studies suggest that antibodies may also have a significant role in HIS [16]. In this study, we assess the protection afforded by DNA vaccines expressing different H1N1 HAs delivered by electroporation against a lethal challenge with seasonal A/New Caledonia/20/99 (H1N1) influenza virus in a mouse model.

\section{Materials and methods}

\subsection{Viruses and cells}

The A/New Caledonia/20/99 (H1N1) influenza virus (GenBank ID: CY033622) was stored in our laboratory. The A/New Caledonia/20/99 (H1N1) influenza virus that had been repeatedly passaged lung-to-lung and adapted in mice was propagated in 10-day-old embryonated specific-pathogen-free chicken eggs at $37^{\circ} \mathrm{C}$ and stored at $-70^{\circ} \mathrm{C}$. Viruses were titrated using the Reed and Muench method to determine the median tissue culture infective dose $\left(\mathrm{TCID}_{50}=\right.$ $10^{-6.25} / 0.05 \mathrm{~mL}$ ) and then used to challenge Balb/c (12 weeks old) mice to determine the median lethal dose $\left(\mathrm{LD}_{50}=\right.$ $10^{-3.5} / 0.05 \mathrm{~mL}$ ). Baby hamster kidney (BHK-21) cells were used for the transient expression experiments. All experiments using the A/New Caledonia/20/99 (H1N1) virus, including the animal challenge experiments, were conducted in accordance with biosafety level 2 containment procedures.

\subsection{Plasmid constructions}

Two different versions of the $H A$ gene (A/New Caledo- nia/20/99 (H1N1), GenBank ID: CY033622 and A/California/07/2009 (H1N1), GenBank ID: GQ117044) were cloned into plasmid vectors. The $H A$ gene of A/New Caledonia/20/99 (H1N1) was obtained by RT-PCR amplification with primers to introduce Nhe I and Xho I restriction sites and a Kozak sequence. The $H A$ gene of A/California/07/ 2009 (H1N1) was commercially synthesized (TaKaRa, Dalian, China). The $H A$ genes of these two strains were ligated into the pVAX1 expression vector (Invitrogen, Carlsbad, CA, USA) at the Nhe I and Xho I restriction sites, resulting in rDNA vector vaccines pV1A5 (A/New Caledonia/20/99 (H1N1)) and pVEH1 (A/California/04/2009 (H1N1)). The nucleotide sequences of both $H A$ genes were confirmed using the ABI PRISM 377XL DNA Sequencer (Applied Biosystems Foster City, CA, USA). The plasmids were propagated in Escherichia coli JM109, and the DNA was extracted using the Plasmid Maxi Kit (Omega Bio-Tek, Doraville, CA, USA). The purified DNA was resuspended in sterile saline solution and stored at $-20^{\circ} \mathrm{C}$ until it was used.

\subsection{Indirect immunofluorescence assay for immuno- genicity}

BHK-21 cells were transfected with purified pV1A5, pVEH1, and pVAX1 DNA using Lipofectamine 2000 (Invitrogen) in accordance with the manufacturer's protocol. Briefly, cell monolayers were grown on glass coverslips in a six-well plate and then transfected with the plasmid DNA $(10 \mu \mathrm{g} /$ well). Forty-eight hours after transfection, the cells were fixed with $0.05 \%$ glutaraldehyde and permeabilized with $0.5 \%$ Triton X-100 in phosphate-buffered saline (PBS), followed by incubation with rabbit polyclonal antibody specific for the HAs of A/New Caledonia/20/99 (H1N1) and A/California/07/2009 (H1N1) (1:2000 in poly(butylene succinate-co-terephthalate) (PBST)) for $1 \mathrm{~h}$ at $37^{\circ} \mathrm{C}$. Fluorescein isothiocyanate (FITC)-conjugated goat anti-rabbit IgG antibodies $(1: 1000)$ with Evans Blue $(1: 200)$ diluted in PBS/bovine serum albumin (BSA) were added and then incubated for $1 \mathrm{~h}$ at room temperature. After mounting the samples, fluorescence images were scanned using an Olympus microscope (BX51; Olympus, Tokyo, Japan).

\subsection{DNA immunization and in vivo electroporation}

Immunization of three groups of six to eight-week-old female Balb/c mice ( $n=15$ in each group) was performed by intramuscular injection of plasmid DNA (100 $\mu \mathrm{g}$ of plasmid DNA in $100 \mu \mathrm{L}$ of PBS ( $\mathrm{pH} 7.4$ ) at two leg sites in the gastrocnemius muscle of each mouse) using a milliliter gauge needle. After injection, a pair of electrode needles $5 \mathrm{~mm}$ apart was inserted into the muscle at the DNA injection site, and electric pulses were delivered using an electric pulse generator (Genetronics, San Diego, CA, USA) [17]. EP 
pulses (three unipolar pulses of $5 \mathrm{~Hz}$ at $75 \mathrm{~V}, 20 \mathrm{~ms}$ each time,) were delivered using an ECM 830 square wave pulse generator (BTX, San Diego, CA, USA). At the end of the third week, each group received one booster immunization identical to the first immunization. Blood samples were taken at one, two, three, and five weeks after primer immunization for determination of antibody titers.

\subsection{Virus challenge in mice}

To assess the efficacy of the two candidate vaccines against lethal infection, the immunized mice were anesthetized two weeks after the second immunization and intranasally challenged with $100 \mathrm{LD}_{50}$ of the A/New Caledonia/20/99 (H1N1) virus in a final volume of $50 \mu \mathrm{L}$. DNA plasmid vectors (pVAX1) lacking an insert were used as a negative control. All studies with the live A/New Caledonia/20/99 (H1N1) virus were performed in accordance with the guidelines of the National Institutes of Health and the Centers for Disease Control and Prevention (www.cdc.gov/flu/h2n2bsl3. $\mathrm{htm}$ ). After infection, the mice were observed every day for $14 \mathrm{~d}$, and survival and clinical parameters such as body weight were recorded. Tissue homogenates were prepared by mechanical disruption of the lungs removed from the dead mice under sterile conditions and used for the RT-PCR assay. The $H A$ gene was amplified and cloned into the pMD18-T vector for sequencing.

\subsection{Enzyme-linked immunosorbent assay}

Samples collected on zero, seven, 14, 21, and $35 \mathrm{~d}$ postimmunization were used to detect IgG antibodies by indirect enzyme-linked immunosorbent assay (ELISA) as previously described [18]. Briefly, 96-well plates (Costar, Cambridge, Mass, USA) were coated with $50 \mathrm{ng}$ of inactivated A/New Caledonia/20/99 (H1N1) virus at $4^{\circ} \mathrm{C}$ overnight, and an ELISA kit for detecting mouse A/California/07/2009 (H1N1) H1HA IgG antibody was used (Cusabio Biotech). Antigen-coated immunoassay plates were washed five times with wash buffer and then blocked with $1 \%$ BSA in PBS for $1 \mathrm{~h}$ at $37^{\circ} \mathrm{C}$. The serum samples were diluted 100 times in PBS containing $0.5 \%$ (w/v) gelatin, $0.15 \%$ Tween 20 , and $4 \%$ calf serum (ELISA diluent) and applied in duplicate wells and incubated for $1 \mathrm{~h}$ at $37^{\circ} \mathrm{C}$. The plates were washed five times with PBS and then reacted with 1:2000 dilutions of HRP-labeled goat anti-mouse IgG (Zhongshan-Golden Bridge Biotech Co., Ltd., Beijing, China) for $1 \mathrm{~h}$ at $37^{\circ} \mathrm{C}$. After another five washes with PBS, the substrate $(10 \mathrm{mg}$ ortho-phenylenediamine $+20 \mathrm{~mL} 0.015 \%$ hydrogen peroxide in phosphate/citrate buffer) was added. After incubation for 15 min at $37^{\circ} \mathrm{C}$, the reactions were terminated with $2 \mathrm{~N} \mathrm{H}_{2} \mathrm{SO}_{4}$. The absorbance values were determined at $492 \mathrm{~nm}$ using a Sunrise automated plate spectrophotometer and analyzed with Microsoft Excel 2007 for Windows. $P$-values were calculated to identify significant differences between the groups.

\subsection{Hemagglutination inhibition test}

Five mice per group were euthanized five weeks after the first immunization. Sera were collected, treated with receptor destroying enzyme (RDE) (Denka-Seiken) and incubated overnight at $37^{\circ} \mathrm{C}$ prior to being tested. Samples were then heat-inactivated at $56^{\circ} \mathrm{C}$ for $30 \mathrm{~min}$. Serum samples were serially diluted two-fold using v-bottom microtiter plates. Equal volumes of $4 \mathrm{HA}$ units of seasonal influenza A/New Caledonia/20/99 (H1N1) virus and A/California/07/ 2009 (H1N1) HA antigen as the source of antigen were added to each well. The plates were mixed by agitation, covered, and allowed to settle for $30 \mathrm{~min}$ at room temperature. Subsequently, $50 \mu \mathrm{L}$ of $1 \%$ chicken red blood cells were added and incubated for $15 \mathrm{~min}$ at $37^{\circ} \mathrm{C}$. The hemagglutination inhibition (HI) titer was determined by reciprocal dilution of the row that contained non-agglutinated chicken red blood cells. Positive and negative serum controls were included in each plate [19].

\subsection{Ex vivo interferon (IFN)- $\gamma$ ELISpot assay}

The level of cellular immunity stimulated by DNA vaccination was evaluated by measuring the frequency of interferon IFN- $\gamma$ secreting splenocytes using a mouse IFN- $\gamma$ ELISpot assay in accordance with the manufacturer's instructions (Dakewe Biotech, China). Dilutions of splenocytes from each mouse $\left(10^{6}\right.$ cells/well $)$ were distributed in polyvinylidene difluoride-treated 96-well plates that were previously coated with an anti-mouse IFN- $\gamma$ monoclonal antibody. Mice splenocytes were added in triplicate with an input cell number of $1 \times 10^{6}$ cells/well in $100 \mu \mathrm{L}$ of 1640 medium with $10 \%$ fetal bovine serum (FBS). The inactivated whole A/New Caledonia/20/99 (H1N1) virus and the A/New Caledonia/20/99 (H1N1) HA antigen were diluted in complete culture medium at a final concentration of $10 \mu \mathrm{g} \mathrm{mL}^{-1}$, and $100 \mu \mathrm{L}$ of the diluted samples was added to each well. Concanavalin A (ConA) $\left(5 \mu \mathrm{g} \mathrm{mL}^{-1}\right)$ was used as a positive control, and cells resuspended in complete culture medium only served as a negative control. After overnight incubation in a $\mathrm{CO}_{2}$ incubator at $37^{\circ} \mathrm{C}$, the splenocytes were discarded from the plates which were then extensively washed with precooled $\mathrm{H}_{2} \mathrm{O}$. The IFN- $\gamma$ spots were detected by biotinylated anti-mouse IFN- $\gamma$ specific antibody. Streptavidin-horseradish peroxidase (HRP) was added and the plates were developed with 3-amino-9-ethylcarbazole (AEC) substrate solution. The spots were counted using an automated ELISpot reader (Bioreader 4000 PRO, Biosys, Germany). The results were expressed as mean \pm SEM of the number of spot-forming cells (SFC) $/ 10^{6}$ spleen cells.

\subsection{Statistical analysis}

The immune response of individual animals was evaluated for statistical analysis. The significance of the cellular and 
humoral immune responses was determined by two-way ANOVA with $P$-values generated using GraphPad 5.0 software. The Mantel-Cox test was used to evaluate the levels of protective immunity between groups. Differences were considered significant when the $P$-value was $<0.05$.

\section{Results}

\subsection{Construction of expression plasmids and the in vitro assessment of immunogenicity}

To determine the immunogenicity of the candidate influenza HA DNA vaccines, plasmids expressing the HAs of A/New Caledonia/20/99 (H1N1) and A/California/07/2009 (H1N1) were constructed and verified by sequencing as described in section 1.2 above. The expression of the HA proteins by pV1A5, pVEH1, and pVAX1 $48 \mathrm{~h}$ after transfection of BHK-21 cells was detected by an immunofluorescence assay with anti-HA serum. The results, shown in Figure 1, indicate that the pV1A5 and pVEH1 plasmids were successfully constructed and express the corresponding HA proteins. Thus, confirming that the plasmids were suitable for use in subsequent experiments.

\subsection{Induction of antibody responses in mice immu- nized with the HA DNA vaccines}

To compare the abilities of the two DNA vaccines to induce HA-specific immune responses, serum samples were obtained from all the mice five weeks after the first immunization and screened for $\mathrm{H} 1 \mathrm{~N} 1 \mathrm{IgG}$ specific antibodies and $\mathrm{HI}$ antibodies against A/New Caledonia/20/99 (H1N1) and A/California/07/2009 (H1N1) HA antigens. HA-specific antibodies were detected in the animals that were immunized with pVEH1 and pV1A5. Remarkably, with four HA units of the A/New Caledonia/20/99 (H1N1) virus, the pV1A5 group mean HI titer was 79, about 12 times higher than the titer of the pVEH1 group (Figure 2A). With four HA units of the A/California/07/2009 (H1N1) HA antigen, the titer of the pV1A5 group was 4.3 times less than that of the pVEH1 group (Figure 2B).
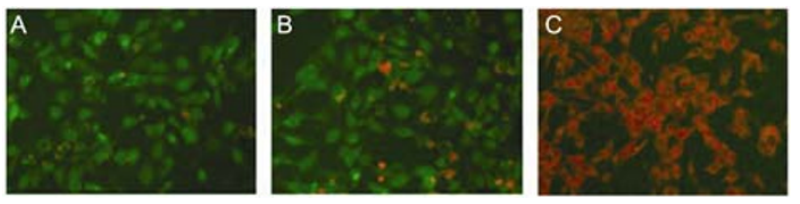

Figure 1 Detection of HA expression by DNA vaccine vectors using indirect immunofluorescence. Green fluorescence of the expressed HA proteins was observed under a fluorescence microscope in BHK-21 cells transfected with pVEH1 (A) or pV1A5 (B). No fluorescence signal was observed in control cells (data not shown) or in BHK-21 cells (C) transfected with pVAX1. To clearly distinguish between cells expressing the HA protein and those that are not, Evans Blue mixed with FITC-conjugated goat anti-rabbit IgG antibodies was used. The red cells are those that do not express the HA protein.
The IgG antibodies were detected using indirect ELISA with the A/New Caledonia/20/99 (H1N1) inactivated whole virus as the coated antigen. The absorbance of samples from mice immunized with pV1A5 rose from $0.27,7 \mathrm{~d}$ post inoculation (7 DPI) to $0.46,14 \mathrm{DPI}$ and then slightly decreased 21 DPI. After receiving the booster dose, the titer rapidly increased to $0.68,35 \mathrm{DPI}$. The mice in the $\mathrm{pVEH} 1$ immunized group only presented mild changes in antibody titers, with the maximum absorbance level corresponding to that of the pV1A5 group 14 DPI. The exception was at 7 DPI when the level of HA-specific IgG antibodies in the pV1A5 DNA vaccine immunized group was higher than that in the pVEH1 group $(P<0.05)$. The titer of the $\mathrm{pVEH} 1$ immunized group was similar to that of the pVAX1 group $(P>0.05)$ except at $35 \mathrm{DPI}$ (Figure $2 \mathrm{C})$. The same response patterns were seen for the antibodies specific for A/California/07/2009 (H1N1) HA antigen and detected by ELISA. The mice in the pVEH1 immunized group showed a significantly higher titer than those in the pV1A5 group $(P<0.05)$ except at 7 DPI (Figure 2D). No specific anti-H1HA antibodies were detected in the mice inoculated with pVAX1. The titer of the pV1A5 immunized group was statistically higher than that of the pVAX1 group $(P<0.05)$ except at $7 \mathrm{DPI}$. These results indicate that, after primary intramuscular vaccination with in vivo electroporation, the DNA vaccine expressing the HA protein significantly induces specific antibody responses at 14 DPI. Furthermore, the IgG antibody levels induced by the two candidate vaccines showed low levels of cross-protection to the heterologous subtype viruses.

\subsection{Cell-mediated immune responses induced in mice immunized with HA DNA vaccines}

Splenocytes were harvested from five immunized mice from each group $3 \mathrm{~d}$ after the boost vaccination. The ELISpot assay was used to assess the magnitude of the HA-specific IFN- $\gamma$ T cell responses after mice were vaccinated with the HA DNA. The harvested splenocytes were stimulated with A/New Caledonia/20/99 (H1N1) inactivated virus and A/California/07/2009 (H1N1) HA antigen for $24 \mathrm{~h}$ and scored in ELISpot assays for IFN- $\gamma$ producing cells. As shown in Figure 3, after stimulation with the inactivated virus samples, the pV1A5 immunized group produced $(353 \pm 18)$ spots $/ 10^{6}$ cells, which was not significantly higher than the $(317 \pm 14)$ spots $/ 10^{6}$ cells produced by the pVEH1 group $(P>0.05)$. Following stimulation with $\mathrm{A} / \mathrm{California} /$ $07 / 2009$ (H1N1) HA antigen the trend was similar, with the pVEH1 immunized group producing $(275 \pm 6)$ spots $/ 10^{6}$ cells and the pV1A5 group producing $(253 \pm 20)$ spots $/ 10^{6}$ cells. A low number of non-specific IFN- $\gamma$ ELISpots were detected in both the control groups $\left(<10 / 10^{6}\right.$ cell spots $)$. The ELISpot background counts in wells containing splenocytes in the absence of nominal antigens were approximately the same as those in the control groups. The positive non-specific 

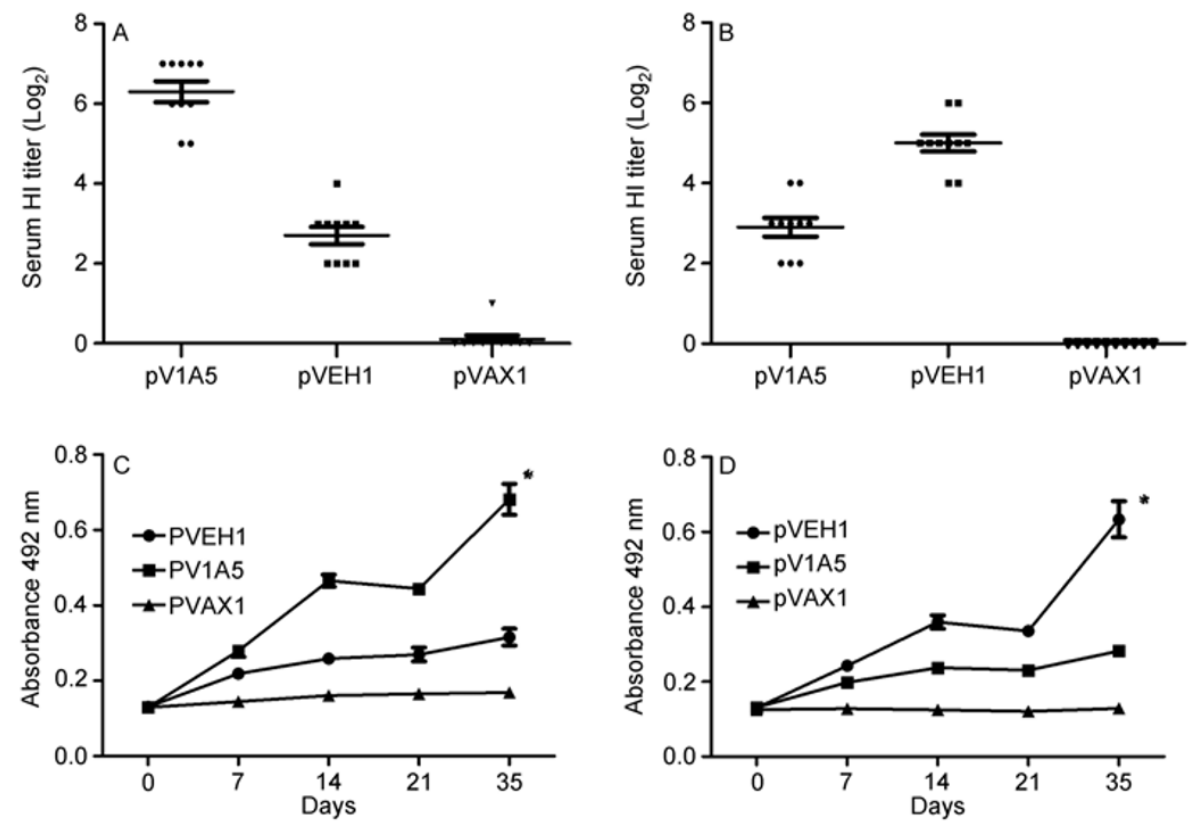

Figure 2 Humoral immunity response of immunized mice. Sera were collected two weeks after the second vaccination and tested individually for HI antibodies against A/New Caledonia/20/99 (H1N1) (A) and A/California/07/2009 (H1N1) HA antigen (B). HI antibody titers for individual mice are expressed as $\log _{2}$ of the reciprocal of the highest dilution of serum inhibiting the agglutination of $1 \%$ chicken erythrocytes by four HA units of the virus or HI antigens. Horizontal lines represent the geometric mean for each group. The IgG specific antibody response after DNA vaccination was measured by indirect ELISA assays that were performed using plates coated with A/New Caledonia/20/99 (H1N1) (C) or A/California/07/2009 (H1N1) HA antigen (D). Sera were collected by tail bleeding zero, one, two, three, and five weeks after the first immunization. Data are shown as mean \pm SEM per group. *, $P<0.05$.

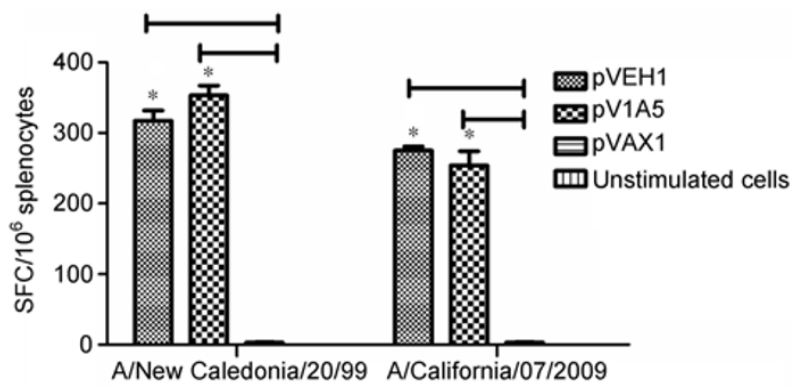

Figure 3 IFN- $\gamma$ secreting splenocytes from immunized mice. Specific responses of the splenocytes $14 \mathrm{~d}$ after the second immunization were determined by an IFN- $\gamma$ ELISpot assay using inactivated whole virus or A/California/07/2009 (H1N1) antigen stimulation. Data are presented as mean \pm SEM per group. SFC, spot forming cells.

IFN- $\gamma$ ELISpot response stimulated by ConA was 300 spots $/ 10^{6}$ cells. Compared with the non-immunized control groups, significant numbers of HA-specific IFN- $\gamma$ ELISpots were detected in all the immunized groups $(P<0.05)$. These results demonstrate that the HA DNA vaccine induced the antigen-specific IFN- $\gamma$ responses. The difference between the ELISpot responses after stimulation with either the A/New Caledonia/20/99 (H1N1) virus or with the A/California/07/2009 (H1N1) HA antigen was not significant $(P>0.05)$.

\subsection{Protective immunity against the virulent H1N1 influenza challenge}

At 35 DPI, three groups of mice were challenged intrana- sally with A/New Caledonia/20/99 (H1N1) and observed for $14 \mathrm{~d}$ for clinical signs and survival rates. All animals immunized with the pVAX1 control experienced substantial weight loss beginning $2 \mathrm{~d}$ post-challenge and were dead 4-8 $\mathrm{d}$ post-challenge. By the end of the experiment, all of the mice in the control group immunized with pVAX1 were dead. By contrast, all animals immunized with pV1A5 showed only mild and transient loss of body weight and all of them survived the lethal challenge. The mice immunized with pVEH1 developed clinical signs such as depression and muscle spasms and began to die $5 \mathrm{~d}$ post-challenge. The mortality rate in the pVEH1 immunized group was $60 \%$. Thus, the protective immunity of the pVEH1 vaccine was significantly lower than that of the pV1A5 vaccine $(P<0.05)$. The percentages of mice that were protected from death or weight loss post-challenge are summarized in Figure 4.

When the sequences of the viruses from the lung supernatants of the pVEH1 and pVAX1 immunized groups were compared, the HA amino acid sequence of A/New Caledonia/20/99 (H1N1) was found in $99.1 \%$ of the pVEH1 group and in $99.7 \%$ of the pVAX1 group. We were unable to amplify an HA positive band from the lung tissue of the mice in the pV1A5 group.

\section{Discussion}

Prophylactic vaccination prior to the epidemic flu season is an efficient way to control influenza virus infections. The currently licensed trivalent inactivated influenza vaccines 

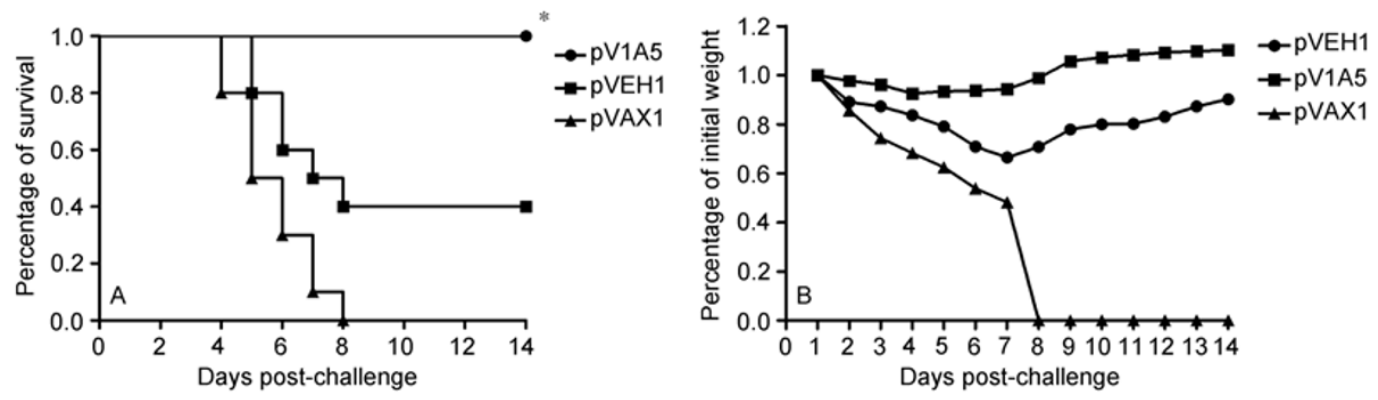

Figure 4 Immune protection conferred by the HA DNA vaccines against the lethal challenge. A, Survival in mice ( $n=10$ mice/group) challenged by intranasal inoculation with $100 \mathrm{LD}_{50}$ of A/New Caledonia/20/99 (H1N1) influenza virus two weeks after the second immunization. *, $P<0.05$. B, Weight loss in the same challenged group of mice. Mean weight loss is expressed as a percentage of their original weight. Data shown are the mean for 10 mice per group.

have proven to be ineffective in conferring protection during epidemic influenza seasons when the vaccine and circulating strains are not well-matched antigenically. To control the pandemic influenza virus, plasmid DNA vaccines have been considered as an alternative to the inactivated vaccines. Previous research has shown that DNA vaccines expressing the H1, H3, H5, H7, and H9 subtypes can protect mice, chickens, and swine against a challenge from a homologous influenza virus [7,8,20-24]. In this study, we investigate whether DNA vaccines can protect against a heterologous viral challenge.

The HA protein is the principal component of the spikes on the viral envelope that mediate the binding of virus particles to cell-surface receptors and trigger subsequent entry into the host cell [25]. Therefore, immunity against the HA antigen can be expected to confer a high level of protection against influenza. Several types of HA-based vaccines have been shown to protect against a challenge from influenza virus of the same HA subtype [26].

In this study, two gene-based DNA vaccines expressing the HA protein of A/New Caledonia/20/99 (H1N1) and A/California/07/2009 (H1N1) were constructed and their abilities to protect against a seasonal A/New Caledonia/20/ 99 (H1N1) challenge in a mouse model were assessed. Consistent with previous research, electroporation-assisted immunization with these two DNA vaccines elicited strong cellular and humoral immune responses to the corresponding virus [27,28]. We initially compared the traditional DNA immunization method with electroporation delivery immunization. The groups that were immunized using electroporation showed significantly higher levels of both humoral and cell-mediated immunity than the groups immunized in the traditional way (data not shown). The humoral immune response of the pV1A5 immunized group was enough to neutralize the A/New Caledonia/20/99 (H1N1) virus challenge, indicating that the antibodies that were produced were necessary and sufficient to confer protective immunity to a lethal challenge with the homologous virus. By contrast, the pVEH1 vaccine encoding the pandemic influenza virus HA protein gave a protective rate of $40 \%$ to the immunized group challenged with the identical seasonal influenza virus. This result indicates that the pVEH1 vaccine did not completely protect the mice against the heterologous subtype challenge. The level of IgG and HI antibodies in this group shows that the heterologous cross-protective immunity was low compared with homologous virus stimulation.

The ELISpot assay was used to assess the magnitude of HA-specific IFN- $\gamma$ T cell responses to stimulation with different antigens. The immunogenic $\mathrm{T}$ cell epitopes may be conserved in the seasonal and pandemic H1N1 influenza viruses because the cell-mediated immune responses to A/New Caledonia/20/99 (H1N1) in the two immunized groups were not significantly different $(P>0.05)$. This result is consistent with a previous report that indicated that half of the predicted CTL epitopes within a swine-origin influenza virus (H1N1) HA are also present in the conventional influenza HA vaccine [29]. The results of the ELISpot assay indicate that, while the humoral immune responses show a significant difference $(P<0.05)$, the levels of cellular cross-protective immunity elicited by the two candidate vaccines are not significantly different $(P>0.05)$. The mean $\mathrm{HI}$ antibody titer of the pVEH1 immunized group against the A/New Caledonia/20/99 (H1N1) antigen was 65 and significantly lower than that of the pV1A5 immunized group with a mean titer of 79 . The same trend was observed for the IgG antibodies. This result may be explained by the $80.1 \%$ amino acid homology between the two H1N1 viral HA proteins, and by antigenic drift that greatly influences the protective immunity against A/New Caledonia/20/99 (H1N1).

Based on our experimental results, we conclude that differences in the humoral immune response may be responsible for the relatively lower protection that was observed against the heterologous subtype challenge. The HA amino acid sequences of the A/California/07/2009 (H1N1) and A/New Caledonia/20/99 (H1N1) viruses differ by $19.9 \%$. As suggested earlier [16], a difference of this magnitude may be enough to qualify as an "antigenic drift", allowing the virus to spread to a pandemic level.

The novelty of the present work is based on two factors. First, we used an electroporation delivery method of immu- 
nization to test our DNA candidate vaccines against influenza. Second, we compared the levels of cross-protective immunity of seasonal and pandemic influenza HA based DNA vaccines in vivo and in vitro by challenges with lethal doses of seasonal influenza virus. Our study validates the utility of electroporation for DNA immunizations and demonstrates the protective efficacy of HA based DNA vaccines against influenza.

This work was supported by the National High Technology Research and Development Program of China (Grant No. 2006AA10A205), the National Key Technology Research and Development Program (Grant No. 2006BAD06A05) and the National Key Program for Infectious Diseases of China (Grant No. 2009ZX10004-103).

1 Fries L F, Dillon S B, Hildreth J E, et al. Safety and immunogenicity of a recombinant protein influenza A vaccine in adult human volunteers and protective efficacy against wild-type H1N1 virus challenge. J Infect Dis, 1993, 167: 593-601

2 Steitz J, Barlow P G, Hossain J, et al. A candidate H1N1 pandemic influenza vaccine elicits protective immunity in mice. PLoS One, 2010, 5: e10492

3 Kong W P, Hood C, Yang Z Y, et al. Protective immunity to lethal challenge of the 1918 pandemic influenza virus by vaccination. Proc Natl Acad Sci USA, 2006, 103: 15987-15991

4 Kodihalli S, Kobasa D L, Webster R G. Strategies for inducing protection against avian influenza A virus subtypes with DNA vaccines. Vaccine, 2000, 18: 2592-2599

5 Kodihalli S, Goto H, Kobasa D L, et al. DNA vaccine encoding hemagglutinin provides protective immunity against H5N1 influenza virus infection in mice. J Virol, 1999, 73: 2094-2098

6 Ljungberg K, Wahren B, Almqvist J, et al. Effective construction of DNA vaccines against variable influenza genes by homologous recombination. Virology, 2000, 268: 244-250

7 Ljungberg K, Kolmskog C, Wahren B, et al. DNA vaccination of ferrets with chimeric influenza A virus hemagglutinin (H3) genes. Vaccine, 2002, 20: 2045-2052

8 Lee C W, Senne D A, Suarez D L. Development of hemagglutinin subtype-specific reference antisera by DNA vaccination of chickens. Avian Dis, 2003, 47: 1051-1056

9 Chen Z, Sahashi Y, Matsuo K, et al. Comparison of the ability of viral protein-expressing plasmid DNAs to protect against influenza. Vaccine, 1998, 16: 1544-1549

10 Chen Z, Kadowaki S, Hagiwara Y, et al. Cross-protection against a lethal influenza virus infection by DNA vaccine to neuraminidase. Vaccine, 2000, 18: 3214-3222

11 Kadowaki S, Chen Z, Asanuma H, et al. Protection against influenza virus infection in mice immunized by administration of hemagglutininexpressing DNAs with electroporation. Vaccine, 2000, 18: 2779-2788

12 Chen Z, Kadowaki S, Hagiwara Y, et al. Protection against influenza $B$ virus infection by immunization with DNA vaccines. Vaccine, 2001, 19: 1446-1455
13 Chen J, Fang F, Li X, et al. Protection against influenza virus infection in BALB/c mice immunized with a single dose of neuraminidaseexpressing DNAs by electroporation. Vaccine, 2005, 23: 4322-4328

14 Luxembourg A, Evans C F, Hannaman D. Electroporation-based DNA immunisation: Translation to the clinic. Expert Opin Biol Ther, 2007, 7: 1647-1664

15 Luckay A, Sidhu M K, Kjeken R, et al. Effect of plasmid DNA vaccine design and in vivo electroporation on the resulting vaccinespecific immune responses in rhesus macaques. J Virol, 2007 81: 5257-5269

16 Nguyen H H, Zemlin M, Ivanov I I, et al. Heterosubtypic immunity to influenza A virus infection requires a properly diversified antibody repertoire. J Virol, 2007, 81: 9331-9338

17 Zhang L, Nolan E, Kreitschitz S, et al. Enhanced delivery of naked DNA to the skin by non-invasive in vivo electroporation. Biochim Biophys Acta, 2002, 1572: 1-9

18 Rowe T, Abernathy R A, Hu-Primmer J, et al. Detection of antibody to avian influenza A (H5N1) virus in human serum by using a combination of serologic assays. J Clin Microbiol, 1999, 37: 937-943

19 Holman D H, Wang D, Raja N U, et al. Multi-antigen vaccines based on complex adenovirus vectors induce protective immune responses against H5N1 avian influenza viruses. Vaccine, 2008, 26: 2627-2639

20 Leili J. Isolation of avian influenza virus and study on construction and immunogenicity of multi-epitopes recombinants. Dissertation for PhD degree. Changchun: Jilin University, 2006

21 Cherbonnel M, Rousset J, Jestin V. Strategies to improve protection against low-pathogenicity $\mathrm{H} 7$ avian influenza virus infection using DNA vaccines. Avian Dis, 2003, 47: 1181-1186

22 Qiu M, Fang F, Chen Y, et al. Protection against avian influenza H9N2 virus challenge by immunization with hemagglutinin- or neuraminidase-expressing DNA in BALB/c mice. Biochem Biophys Res Commun, 2006, 343: 1124-1131

23 Laddy D J, Yan J, Corbitt N, et al. Immunogenicity of novel consensus-based DNA vaccines against avian influenza. Vaccine, 2007, 25: 2984-2989

24 Wenlong N. Screening of influenza functional epitopes and study on design and immunogenicity of combined multi-epitope DNA vaccine. Dissertation for PhD degree. Changchun: Jilin University, 2009

25 Carroll S M, Paulson J C. Differential infection of receptor-modified host cells by receptor-specific influenza viruses. Virus Res, 1985, 3 : 165-179

26 Wang S X, Taaffe J, Parker C, et al. Hemagglutinin (HA) proteins from $\mathrm{H} 1$ and $\mathrm{H} 3$ serotypes of influenza $\mathrm{A}$ viruses require different antigen designs for the induction of optimal protective antibody responses as studied by codon-optimized HA DNA vaccines. J Virol, 2006, 80: 11628-11637

27 Zheng L Y, Wang F Y, Yang Z D, et al. A single immunization with HA DNA vaccine by electroporation induces early protection against $\mathrm{H} 5 \mathrm{~N} 1$ avian influenza virus challenge in mice. BMC Infect Dis, 2009, 9: 17

28 Zhang L, Widera G, Rabussay D. Enhancement of the effectiveness of electroporation-augmented cutaneous DNA vaccination by a particulate adjuvant. Bioelectrochem, 2004, 63: 369-373

29 De Groot A S, Arditoa M, McClaine E M, et al. Immunoinformatic comparison of $\mathrm{T}$-cell epitopes contained in novel swine-origin influenza A (H1N1) virus with epitopes in 2008-2009 conventional influenza vaccine. Vaccine, 2009, 27: 5740-5747

Open Access This article is distributed under the terms of the Creative Commons Attribution License which permits any use, distribution, and reproduction in any medium, provided the original author(s) and source are credited. 